\title{
The Impact of Triglyceride-Glucose Index On Incident Cardiovascular Events in Patients With Type 2 Diabetes Mellitus
}

\section{Liyao Fu}

The Second Xiangya Hospital of Central South University

Shi Tai ( $\nabla$ taishi2017@csu.edu.cn )

The Second Xiangaya Hospital of Central South University https://orcid.org/0000-0002-5802-2910

Jiaxing Sun

Second Xiangya Hospital

\section{Ningjie Zhang}

Second Xiangya Hospital

Ying Zhou

Second Xiangya Hospital

\section{Zhenhua Xing}

Second Xiangya Hospital

Yongjun Wang

Second Xiangya Hospital

\section{Shenghua Zhou}

Second Xiangya Hospital

\section{Original investigation}

Keywords: Triglyceride-glucose index, Cardiovascular disease, Type 2 diabetes mellitus, Risk factors, Insulin resistance

Posted Date: October 12th, 2021

DOI: https://doi.org/10.21203/rs.3.rs-960363/v1

License: (c) (i) This work is licensed under a Creative Commons Attribution 4.0 International License. Read Full License 


\section{Abstract}

Background: Previous studies reported the prognostic value of the triglyceride-glucose (TyG) index in the course of cardiovascular (CV) diseases. Still, it remains unclear whether baseline and trajectories of TyG index are prospectively associated with incident CV events among patients with type 2 diabetes mellitus (T2DM).

Methods: We performed a secondary analysis in patients with long-lasting T2DM from the Action to Control Cardiovascular Risk in Diabetes (ACCORD) study. The primary outcome was the first occurrence of adverse CV events including nonfatal myocardial infarction, nonfatal stroke, or death from cardiovascular causes, and the TyG index was measured at 11 visits. Cox proportional hazards regression analysis was used to observe the association between baseline and trajectories of TyG index and adverse CV outcomes.

Results: During a median follow-up period of 8.8 years, 1,815 (17.8\%) developed at least one primary endpoint event. After adjusting for traditional cardiovascular risk factors, each 1-SD increase in the TyG index was associated with a $19.00 \%$ higher risk of adverse CV events, similar in individuals categorized by TyG index quartiles. Four distinct trajectories of TyG indexes were identified- low (16.17\%), moderate (40.01\%), high (34.60\%), and very high (9.30\%). Among these, moderate, high, and very high TyG index trajectories had a greater risk of future incident adverse CV events than low TyG index trajectories after multivariate adjustments for traditional risk factors. Particularly, a similar association was noticed in the TyG index and the occurrence of coronary heart disease.

Conclusions: The findings of this study suggest that both baseline and trajectories of TyG index have a significant association with the occurrence of adverse CV events in patients with T2DM.

(Trial registration: URL: http://www.clinicaltrials.gov. Unique identifier: NCT00000620)

\section{Introduction}

Type 2 diabetes mellitus (T2DM) has been associated with the early onset of atherosclerotic cardiovascular disease (CVD), often presenting as coronary heart disease (CHD), cerebrovascular disease, and peripheral arterial disease (PAD) of atherosclerotic origin in patients[1]. Specifically, diabetic patients typically develop cardiovascular (CV) abnormality with greater severity 14.6 years in advance compared to individuals without diabetes mellitus (DM)[2, 3]. Established risk factors for CVD include hypertension (HTN) and dyslipidemia, which are common in patients with T2DM[1]. Studies showed that patients preconditioned with dyslipidemia had dysregulated lipid and glucose metabolism, such as insulin resistance (IR), resulting in a poorer prognosis of CVD[4]. The incidences of T2DM complications have reduced over the years due to advances in medicine. However, more than 382 million people in the world currently have diabetes, making them more vulnerable to CVD-related disability and deaths[5]. Therefore, there is an urgent need for CVD prevention in diabetic individuals. To achieve this goal, it is necessary to develop effective ways to predict and diagnose T2DM-related CVD more accurate at the early stages.

Correspondingly, many studies indicated that IR contributes significantly to the development of atherosclerotic CVD[6]. The underlying mechanism that IR and coexisting hyperinsulinemia are implicated in developing dyslipidemia, HTN, hypercoagulability, and atherosclerosis[7, 8]. The homeostasis model assessment of insulin resistance (HOMA-IR) index is the most common method for evaluating IR in clinical practice[9]. However, this method of assessing IR is complex and expensive[10]. Therefore, several surrogate markers of IR have been proposed and compared with the gold standard of the hyperinsulinemic-euglycemic clamp[11]. The triglyceride-glucose (TyG) index, which is calculated using fasting triglycerides (TG) and fasting glucose, is a reliable measure of IR $[12,13]$. The TyG index has been proved to be highly correlated with the euglycemic-hyperinsulinemic clamp test [12]. It thus has a validity similar to HOMA-IR [14]. Growing evidence has demonstrated that the TyG index is related to morbidity and mortality of cardiovascular disease in the general population[15] and predicts progression of coronary artery calcification[16]. 
However, the role of TyG in patients with T2DM has been inadequately explored compared with those without T2DM. Previous studies evaluating the association of the TyG index with a predictor of CVD and adverse CV outcomes are inherently limited by small sample sizes, a retrospective study, or a cross-sectional analysis[15, 17-22]. Notably, the TyG index at a single time point or baseline TyG was used for relationship analysis. To our best knowledge, few long-term prospective studies have been performed to explore the relationship between adverse CV outcomes and the TyG index in patients with T2DM and its trajectory derived from the multiple measurements over time. Patients with T2DM with glycosylated hemoglobin ( $\mathrm{HbA1c}$ ) concentration of at least $7.5 \%$ and a history of CVD indicated by the anatomical evidence of significant atherosclerosis, albuminuria, left ventricular hypertrophy, or at least two risk factors for CVDs, which were included in this study. Besides, tight control of both blood pressure and lipids also did not reduce the chance of CVD. Moreover, the intensive glycemic intervention was terminated in this study after a mean follow-up of 3.7 years, owing to increased mortality in the intensive glycemic control group. All participants were transitioned to the standard glycemic control intervention[23, 24].

Furthermore, in this study, group-based trajectory analysis was designed to identify clusters of individuals with similar patterns of change over time [25]. Participants were assigned to the trajectory group for which they had the greatest posterior predictive probability. Accordingly, we hypothesized that dynamic changes in TyG during follow-up might modify the development of adverse CV outcomes. In this large-scale study, to evaluate the association of the TyG index with adverse CV outcomes and to determine the influence of baseline TyG index and different trajectories of its change over 10 years on the development of adverse CV outcomes, the data from the action to control CV risk in diabetes (ACCORD)[23] study and ACCORD follow-on study (ACCORDION)[26] were used accordingly. Taken together, this study aimed to evaluate the impact of the TyG index on incident CV events in patients with T2DM during long-term follow-up.

\section{Methods}

\section{Study design and subjects}

In this study, a secondary analysis of 10,251 T2DM patients from published data of the ACCORD/ACCORDION trial (ClinicalTrials.gov number, NCT00000620) was performed [27]. Briefly, ACCORD was a $2 \times 2$ factorial trial aimed to test whether strict control of blood glucose, blood pressure, and lipids could reduce CVD incidence in T2DM patients. The rationale and design of the ACCORD trial have been described previously. All the subjects recruited from 77 clinical sites across North America between January 2001 to October 2005. The study follow-up gave in the ACCORD trial, and closeout visits were completed by June 2009. At the final trial visits, the study participants were invited to participate in the post-trial, nontreatment, observation-only ACCORDION study. The study follow-up ended 60 months post ACCORD (October 31, 2014) for post-trial observation.

The inclusion criteria: 1) patients who had type 2 diabetes mellitus and a glycated hemoglobin level of $7.5 \%$ or more; 2 ) who either were between the ages of 40 and 79 years and had cardiovascular disease; 3 ) who were between the ages of 55 and 79 years and had anatomical evidence of significant atherosclerosis, albuminuria, left ventricular hypertrophy; 4) at least two additional risk factors for cardiovascular disease (dyslipidemia, hypertension, current status as a smoker, or obesity). The exclusion criteria: 1) frequent or recent serious hypoglycemic events; 2) unwillingness to do home glucose monitoring or inject insulin; 3) a body-mass index (the weight in kilograms divided by the square of the height in meters) of more than 45; 4) a serum creatinine level of more than $1.5 \mathrm{mg}$ per deciliter (133 $\mu \mathrm{mol}$ per liter); 5$)$ other serious illness.

This ACCORD/ACCORDION study was approved by the Wake Forest University, USA (coordinating center), and the institutional review boards (IRB) at each center (participating clinical sites). Written informed consent was obtained from all ACCORD/ACCORDION participants.

\section{Exclusion criteria of this study}


The participants who had missing data regarding TyG index values at baseline $(n=55)$ were excluded from our study. This resulted in a final sample of 10196 participants to analyze the association between baseline TyG index and primary and secondary outcomes. Those participants with fewer than three valid TyG indexes during follow-up visits were also excluded. The remaining 9697 participants were included in the analysis of the association between TyG index group-based trajectory and adverse CV outcomes (Fig. 1).

\section{Outcomes}

The primary outcome of the study was major adverse cardiovascular events, including non-fatal myocardial infarction (MI), non-fatal stroke, and death from cardiovascular causes. Secondary outcomes were all-cause mortality, total stroke, congestive heart failure (CHF), and major coronary events (CV death, non-fatal MI, or unstable angina). Participants were followed up every 2-4 months via phone interviews or visits at the outpatient clinic. Relevant medical information was collected during each follow-up. The first occurrence of adverse cardiovascular events in each patient was determined by a Working Group of the Morbidity and Mortality subcommittee. In addition, the major adverse cardiovascular events were also collected with follow-up ended on October 31, 2014, or 60 months after ACCORD, all patients for a total of 5 years of posttrial observation.

\section{Data collection}

The data included patients' demographic and clinical characteristics, age, gender, ethnicity, education, smoking history, medical history, previous medications, body measurements, blood content (i.e., fasting glucose, fasting plasma triglycerides, cholesterol, LDL-C, and HDL-C), etc. was collected. The fasting glucose and fasting triglycerides were further collected during follow-up visits. The TyG index was calculated as In (fasting TG [mg/dL] × fasting glucose [mg/dL]/2) [12].

\section{Statistical analysis}

Normally distributed continuous data were expressed as mean \pm SD, non-normally distributed continuous data were expressed as median (interquartile range), and categorical data were expressed as numbers (percentage). Differences among groups were evaluated using analysis of variance (ANOVA) or Kruskal-Wallis h-test when appropriate for the continuous variables and the $\chi 2$ test for the categorical variables. Kaplan-Meier estimates were used to compute the cumulative incidence of incident adverse CV events by TyG index quartiles. The differences in estimates were compared using the log-rank procedure. Cox proportional hazards regression model was used to calculate hazard ratios and $95 \% \mathrm{Cls}$ between TyG index and time for adverse CV event. Three multivariate models with progressive degrees of adjustment were used to adjust for potential confounders of an adverse CV event. These models are- Model 1: adjusted for baseline age, sex, previous CV event, race, BMI, education, systolic blood pressure, and diastolic blood pressure; Model 2: adjusted for model 1 covariates plus baseline eGFR, HbA1c, total plasma cholesterol, plasma HDL-C, and depression; and Model 3: adjusted for model 2 covariates plus statin, biguanide, aspirin, ACEI/ARB, and insulin.

Further, we used a restricted cubic spline regression model with 3 knots to assess the nonlinear dose-response association between baseline TyG index and adverse CV events. We used latent class models to identify different longitudinal TyG index levels patterns within the ACCORD study participants and tested models with groups ranging from 2 to 5 . We examined different criteria, including Bayesian information criteria, to assess the optimal number of trajectories. No group included less than $5 \%$ of participants (see Table S1). All the final models classified participants into trajectory groups with good discrimination: the mean probability of final group membership was 0.89 . The trajectory group was included as an independent variable in a Cox proportional hazards regression model examining predictors of adverse CV events at a followup to estimate the association of TyG index trajectory groups with adverse CV events. All analyses were conducted in SPSS version 23 (SPSS, Inc, Chicago, Illinois) and Stata 15.1 (Stata Corp LLC, Texas, USA). A two-sided P value of $<0.05$ was considered statistically significant.

\section{Results}




\section{Baseline characteristics according to quartiles of TyG index}

Analysis included 10,196 participants at baseline, $61.48 \%$ male with a mean age of $62.77 \pm 6.63$ years. The mean TyG index was $9.49 \pm 0.73$ (Table 1). We categorized the included population into four groups based on the quartiles of the baseline TyG index (Table 1). Participants with a higher TyG index were young and more often male and white. They had higher levels of BMI, DBP, TC, LDL-C, TG, fasting glucose, glycated hemoglobin, eGFR, serum creatinine, lower levels of education, and less frequently current drinkers (all $p<0.001$ ). Likewise, participants in a higher TyG index quartile had a higher prevalence of cardiovascular disease and congestive heart failure. They were more prone to take metformin drugs and less prone to take insulin, ACEI/ARB, statin, cholesterol absorption inhibitors (all $p<0.001$ ). 
Table 1

Baseline characteristics of participants by quartiles of TyG index

\begin{tabular}{|c|c|c|c|c|c|c|}
\hline & $\begin{array}{l}\text { Total } \\
(n=10,196)\end{array}$ & $\begin{array}{l}\text { Q1 } \\
\text { (Minimum) } \\
(n=2,550)\end{array}$ & $\begin{array}{l}\text { Q2 } \\
(n=2,550)\end{array}$ & $\begin{array}{l}\text { Q3 } \\
(n=2,547)\end{array}$ & $\begin{array}{l}\text { Q4 } \\
\text { (Maximum) } \\
(n=2,549)\end{array}$ & $\begin{array}{l}p \\
\text { value }\end{array}$ \\
\hline Age, y $($ mean $\pm S D)$ & $62.77 \pm 6.63$ & $63.512 \pm 6.91$ & $63.24 \pm 6.61$ & $62.72 \pm 6.58$ & $61.57 \pm 6.24$ & $<0.001$ \\
\hline TyG index & $9.49 \pm 0.73$ & $8.58 \pm 0.35$ & $9.25 \pm 0.13$ & $9.70 \pm 0.14$ & $10.42 \pm 0.43$ & $<0.001$ \\
\hline $\operatorname{Sex}[n(\%)]$ & & & & & & $<0.001$ \\
\hline Female & $3,928(38.52 \%)$ & $1,000(39.22 \%)$ & $990(38.82 \%)$ & $988(38.79 \%)$ & $950(37.27 \%)$ & \\
\hline Male & $6,268(61.48 \%)$ & $1,550(60.78 \%)$ & $1,560(61.18 \%)$ & $\begin{array}{l}1,559 \\
(61.21 \%)\end{array}$ & $1,599(62.73 \%)$ & \\
\hline $\begin{array}{l}\text { Race/ethnicity, } \\
{[n(\%)]}\end{array}$ & & & & & & $<0.001$ \\
\hline White & $6,371(62.49 \%)$ & $1,162(45.57 \%)$ & $1,530(60.00 \%)$ & $1,775(69.69 \%)$ & $1,904(74.70)$ & \\
\hline Non-White & $3,825(60.04)$ & $1,388(53.43 \%)$ & $1,020(40.00 \%)$ & $772(30.31 \%)$ & $645(25.30 \%)$ & \\
\hline \multicolumn{7}{|l|}{ Education, [n(\%)] } \\
\hline $\begin{array}{l}\text { Less than high } \\
\text { school(1) }\end{array}$ & $1,502(14.73 \%)$ & $429(16.82 \%)$ & $407(15.96 \%)$ & $350(13.74 \%)$ & $316(12.40 \%)$ & $<0.001$ \\
\hline $\begin{array}{l}\text { High school } \\
\text { graduate or GED }\end{array}$ & $2,692(26.40 \%)$ & $713(27.96 \%)$ & $649(25.45 \%)$ & $670(26.31 \%)$ & $660(25.89 \%)$ & $<0.001$ \\
\hline Some college & $3,343(32.79 \%)$ & $761(29.84 \%)$ & $812(31.84 \%)$ & $868(34.08 \%)$ & $902(35.39 \%)$ & $<0.001$ \\
\hline $\begin{array}{l}\text { College degree or } \\
\text { higher }\end{array}$ & $2,652(26.01 \%)$ & $644(25.25 \%)$ & $679(26.63 \%)$ & $658(25.83 \%)$ & $671(26.32 \%)$ & $<0.001$ \\
\hline $\begin{array}{l}\text { Previous } \\
\text { cardiovascular } \\
\text { event, [n(\%)] }\end{array}$ & $3,586(35.17 \%)$ & $855(33.53 \%)$ & $878(34.43 \%)$ & $896(35.18 \%)$ & $957(37.54 \%)$ & 0.019 \\
\hline $\begin{array}{l}\text { Previous } \\
\text { congestive heart } \\
\text { failure, }[n(\%)]\end{array}$ & $489(4.80 \%)$ & $105(4.12 \%)$ & $107(4.20 \%)$ & $127(4.99 \%)$ & $150(5.88 \%)$ & 0.01 \\
\hline $\begin{array}{l}\text { Duration of } \\
\text { diabetes, y (mean } \\
\pm \text { SD) }\end{array}$ & $10.80 \pm 7.60$ & $12.22 \pm 8.24$ & $10.96 \pm 7.61$ & $10.26 \pm 7.34$ & $9.77 \pm 6.91$ & $<0.001$ \\
\hline Live alone, [n(\%)] & $2,065(20.65 \%)$ & $533(20.90 \%)$ & 499(19.57\%) & $531(20.85 \%)$ & $502(19.69 \%)$ & 0.484 \\
\hline Depression, [n(\%)] & $2,412(23.66 \%)$ & $475(18.63 \%)$ & $538(21.10 \%)$ & $653(25.64 \%)$ & $746(29.27 \%)$ & $<0.001$ \\
\hline $\begin{array}{l}\text { Cigarette-smoking } \\
\text { status, }[\mathrm{n}(\%)]\end{array}$ & & & & & & $<0.001$ \\
\hline Yes & $5,925(58.11 \%)$ & $1401(54.94 \%)$ & $1467(57.53)$ & $1513(59.40 \%)$ & $1544(60.57 \%)$ & \\
\hline
\end{tabular}

Quartile ranges are Q1 (6.87 to 9.00), Q2(9.01 to 9.47), Q3(9.47 to 9.95), and Q4 (9.95 to 13.36).

$P$ value for test of difference across quartiles of triglyceride-glucose (TyG) index using chi-square test (categorical variables) or analysis of variance (continuous variables) or Kruskal-Wallis test (nonparametric comparisons)

eGFR: estimated glomerular filtration rate; LDL-C: low-density lipoprotein cholesterol; ACEl: angiotensin-converting enzyme inhibitor; ARB: angiotensin receptor blocker. 


\begin{tabular}{|c|c|c|c|c|c|c|}
\hline & $\begin{array}{l}\text { Total } \\
(n=10,196)\end{array}$ & $\begin{array}{l}\text { Q1 } \\
\text { (Minimum) } \\
(n=2,550)\end{array}$ & $\begin{array}{l}\text { Q2 } \\
(n=2,550)\end{array}$ & $\begin{array}{l}\text { Q3 } \\
(n=2,547)\end{array}$ & $\begin{array}{l}\text { Q4 } \\
\text { (Maximum) } \\
(n=2,549)\end{array}$ & $\begin{array}{l}p \\
\text { value }\end{array}$ \\
\hline No & $4,271(41.89 \%)$ & $1149(45.06 \%)$ & 1083(42.47\%) & 1034(40.60) & 1005(39.43\%) & \\
\hline $\begin{array}{l}\text { Alcohol status, } \\
\text { [n(\%)] }\end{array}$ & & & & & & $<0.001$ \\
\hline Yes & $2,434(23.87 \%)$ & $556(21.80 \%)$ & $636(24.94 \%)$ & $626(24.58 \%)$ & 616(24.17\%) & \\
\hline No & 7,757(76.08\%) & 1993(78.16\%) & 1912(74.98\%) & 1920(75.38\%) & $1930(75.72 \%)$ & \\
\hline $\begin{array}{l}\text { Weight, kg (mean } \pm \\
\text { SD) }\end{array}$ & $93.51 \pm 18.41$ & $89.94 \pm 18.42$ & $93.26 \pm 18.11$ & $94.69 \pm 18.36$ & $96.29 \pm 18.13$ & $<0.001$ \\
\hline $\begin{array}{l}\text { Waist } \\
\text { circumference, cm } \\
\text { (mean } \pm \text { SD) }\end{array}$ & $106.73 \pm 13.64$ & $103.63 \pm 13.96$ & $106.59 \pm 13.48$ & $107.79 \pm 13.35$ & $108.94 \pm 13.17$ & $<0.001$ \\
\hline $\begin{array}{l}\text { Body mass index, } \\
\mathrm{kg} / \mathrm{m}^{2} \text { (mean } \pm \\
\mathrm{SD})\end{array}$ & $32.22 \pm 5.42$ & $31.15 \pm 5.56$ & $32.18 \pm 5.41$ & $32.60 \pm 5.31$ & $32.96 \pm 5.22$ & $<0.001$ \\
\hline \multicolumn{7}{|l|}{$\begin{array}{l}\text { Blood pressure, } \\
\text { mmHg (mean } \pm \\
\text { SD) }\end{array}$} \\
\hline Systolic & $136.36 \pm 17.11$ & $136.79 \pm 17.22$ & $136.16 \pm 16.83$ & $135.52 \pm 17.37$ & $136.92 \pm 16.99$ & 0.0125 \\
\hline Diastolic & $74.88 \pm 10.66$ & $73.63 \pm 10.61$ & $74.40 \pm 10.47$ & $75.10 \pm 10.57$ & $76.40 \pm 10.81$ & $<0.001$ \\
\hline \multicolumn{7}{|l|}{ Medications, [n(\%)] } \\
\hline Insulin & $3,565(34.96 \%)$ & $1,121(43.96 \%)$ & $935(36.67 \%)$ & $760(29.84 \%)$ & 749 (29.38\%) & $<0.001$ \\
\hline Metformin & 6,519(63.94\%) & $1,550(60.78 \%)$ & $1,648(64.63 \%)$ & 1,695(66.55\%) & $1,626(66.79 \%)$ & $<0.001$ \\
\hline ACEI/ARB & $7,066(69.30 \%)$ & $1,804(70.75 \%)$ & $1,810(70.98 \%)$ & $1,757(68.98 \%)$ & $1,695(66.50 \%)$ & 0.001 \\
\hline Aspirin & $5,552(54.45 \%)$ & $1,412(55.37 \%)$ & $1,421(55.73 \%)$ & 1,384(54.34\%) & 1,335 (52.37\%) & 0.072 \\
\hline Statin & $6,468(63.44 \%)$ & $1,732(67.92 \%)$ & $1,681(65.92 \%)$ & $1,618(63.53 \%)$ & $1,437(56.38 \%)$ & $<0.001$ \\
\hline $\begin{array}{l}\text { Cholesterol } \\
\text { absorption } \\
\text { inhibitors }\end{array}$ & $207(2.03 \%)$ & $46(1.80 \%)$ & $53(2.08 \%)$ & $63(2.47 \%)$ & $45(1.77 \%)$ & 0.251 \\
\hline $\begin{array}{l}\text { Glycated } \\
\text { hemoglobin, \% } \\
\text { (mean } \pm \text { SD) }\end{array}$ & $8.30 \pm 1.06$ & $8.04 \pm 0.96$ & $8.15 \pm 0.96$ & $8.34 \pm 1.03$ & $8.67 \pm 1.15$ & $<0.001$ \\
\hline $\begin{array}{l}\text { Fasting plasma } \\
\text { glucose, } \mathrm{mg} / \mathrm{dL} \\
(\text { mean } \pm \mathrm{SD})\end{array}$ & $175.19 \pm 56.17$ & $133.00 \pm 38.86$ & $164.79 \pm 42.02$ & $183.60 \pm 44.72$ & $219.43 \pm 58.59$ & $<0.001$ \\
\hline $\begin{array}{l}\text { Potassium, } \mathrm{mg} / \mathrm{dL} \\
(\text { mean } \pm \text { SD) }\end{array}$ & $4.47 \pm 0.47$ & $4.43 \pm .46$ & $4.47 \pm 0.43$ & $4.50 \pm 0.54$ & $4.50 \pm 0.44$ & $<0.001$ \\
\hline \multicolumn{7}{|c|}{ Quartile ranges are Q1 (6.87 to 9.00), Q2(9.01 to 9.47), Q3(9.47 to 9.95), and Q4 (9.95 to 13.36). } \\
\hline \multicolumn{7}{|c|}{$\begin{array}{l}P \text { value for test of difference across quartiles of triglyceride-glucose (TyG) index using chi-square test (categorical } \\
\text { variables) or analysis of variance (continuous variables) or Kruskal-Wallis test (nonparametric comparisons) }\end{array}$} \\
\hline
\end{tabular}




\begin{tabular}{|c|c|c|c|c|c|c|}
\hline & $\begin{array}{l}\text { Total } \\
(n=10,196)\end{array}$ & $\begin{array}{l}\text { Q1 } \\
\text { (Minimum) } \\
(n=2,550)\end{array}$ & $\begin{array}{l}\text { Q2 } \\
(n=2,550)\end{array}$ & $\begin{array}{l}\text { Q3 } \\
(n=2,547)\end{array}$ & $\begin{array}{l}\text { Q4 } \\
\text { (Maximum) } \\
(n=2,549)\end{array}$ & $\begin{array}{l}p \\
\text { value }\end{array}$ \\
\hline $\begin{array}{l}\text { Serum creatinine, } \\
\mathrm{mg} / \mathrm{dL} \text { (mean } \pm \\
\mathrm{SD} \text { ) }\end{array}$ & $0.91 \pm 0.23$ & $0.93 \pm 0.23$ & $0.92 \pm 0.23$ & $0.91 \pm 0.23$ & $0.89 \pm 0.24$ & $<0.001$ \\
\hline $\begin{array}{l}\text { eGFR, } \\
\left(\mathrm{mL} / \mathrm{min} / 1.73 \mathrm{~m}^{2}\right) \\
(\mathrm{mean} \pm \mathrm{SD})\end{array}$ & $91.05 \pm 27.15$ & $91.11 \pm 24.21$ & $90.58 \pm 25.44$ & $89.54 \pm 24.66$ & $92.93 \pm 33.19$ & $<0.001$ \\
\hline $\begin{array}{l}\text { Plasma } \\
\text { triglycerides, } \\
\text { mg/dL (mean } \pm \\
\text { SD) }\end{array}$ & $190.11 \pm 148.40$ & $88.57 \pm 29.18$ & $134.60 \pm 36.89$ & $188.78 \pm 49.37$ & $348.62 \pm 211.93$ & $<0.001$ \\
\hline $\begin{array}{l}\text { Total plasma } \\
\text { cholesterol, mg/dL } \\
\text { (mean } \pm \text { SD) }\end{array}$ & $183.29 \pm 41.89$ & $166.51 \pm 34.40$ & $175.85 \pm 35.50$ & $184.97 \pm 37.50$ & $205.91 \pm 205.9$ & $<0.001$ \\
\hline $\begin{array}{l}\text { Plasma LDL-C, } \\
\mathrm{mg} / \mathrm{dL} \text { (mean } \pm \\
\mathrm{SD} \text { ) }\end{array}$ & $104.89 \pm 33.93$ & $100.67 \pm 29.79$ & $105.63 \pm 31.92$ & $107.25 \pm 34.12$ & $106.04 \pm 38.80$ & $<0.001$ \\
\hline $\begin{array}{l}\text { Incident adverse } \\
\text { cardiovascular } \\
\text { events, [n(\%)] }\end{array}$ & $1815(17.80 \%)$ & $389(15.25 \%)$ & $429(16.82 \%)$ & $460(18.06 \% \%)$ & $537(21.07 \%)$ & $<0.001$ \\
\hline \multicolumn{7}{|c|}{ Quartile ranges are Q1 (6.87 to 9.00), Q2(9.01 to 9.47), Q3(9.47 to 9.95), and Q4 (9.95 to 13.36). } \\
\hline \multicolumn{7}{|c|}{$\begin{array}{l}P \text { value for test of difference across quartiles of triglyceride-glucose (TyG) index using chi-square test (categorical } \\
\text { variables) or analysis of variance (continuous variables) or Kruskal-Wallis test (nonparametric comparisons) }\end{array}$} \\
\hline
\end{tabular}

\section{Associations between baseline TyG index and primary and secondary adverse CV outcomes}

During a median of 8.8 years and a mean of 7.7 years from randomization, 1815 patients $(17.8 \%)$ developed primary endpoint event. As Table 1 shows, the risk of adverse CV event increased with increasing quartiles of TyG index [quartiles 14: 389 (15.25\%) vs. 429 (16.82\%) vs. 460 (18.06\%) vs. 537 (21.07\%); P < 0.001]. In the multivariate model that measured TyG index as a continuous variable, a 1-SD increase in the TyG index was associated with a $19.00 \%$ higher risk of adverse CV events after full adjustment for the potential confounders (HR 1.19, 95\% Cl $1.11-1.28 ; p<0.001$; Table 2). Results were similar when we categorized individuals by TyG index quartiles: the highest risk of adverse CV events was observed in the participants with the highest TyG index quartile, in 3 different adjusted models (all $p<0.05$, Table 2). In the final model, the hazard ratios (95\% Cls) for adverse CV events comparing the fourth quartile of TyG index with the first quartile was 1.13 (95\% Cl, 1.06-1.20) (model 3 in Table 2; Fig. 2). Results were similar when evaluated association between baseline TyG index and all-cause death, CV death, non-fatal MI, non-fatal stroke, total stroke, and fatal or hospital congestive heart failure (Table S2). Notably, the HR $(95 \% \mathrm{Cls})$ in the final model, for major coronary events comparing the second, third, fourth quartile of TyG index with the first quartile were 1.22(95\% Cl, 1.06-1.41), 1.12(95\% Cl, 1.03-1.22), and 1.17(95\% Cl, 1.10-1.24), respectively, (all $p<0.05$, model 3 in Table 2; Fig. 2). These findings suggested that baseline TyG index significantly was correlated with major coronary events, including CV death, non-fatal MI, or unstable angina. Figure 3 shows the restricted cubic splines of the risk of adverse CV events across levels of the TyG index. Consistent with the analysis using quartiles of sample distribution, the risk of incident adverse CV events increased in participants with a higher TyG index (Fig. 3). 
Table 2. Risk of incident adverse CV and major coronary events for baseline TyG index Adverse CV events (a composite of CV death, non-fatal Ml, or non-fatal stroke)

\begin{tabular}{|c|c|c|c|c|c|c|c|c|c|}
\hline $\begin{array}{l}\text { TyG } \\
\text { index }\end{array}$ & $\begin{array}{l}\text { Events/No. } \\
\text { at risk }\end{array}$ & $\begin{array}{l}\text { Unadjusted } \\
\text { HR }(95 \% \mathrm{Cl})\end{array}$ & $\begin{array}{l}p \\
\text { value }\end{array}$ & $\begin{array}{l}\text { Model } 1 \\
\text { HR (95\% } \\
\text { Cl) }\end{array}$ & $\begin{array}{l}p \\
\text { value }\end{array}$ & $\begin{array}{l}\text { Model } 2 \\
\text { HR }(95 \% \\
\text { Cl) }\end{array}$ & $\begin{array}{l}p \\
\text { value }\end{array}$ & $\begin{array}{l}\text { Model } 3 \\
\text { HR }(95 \% \\
\mathrm{Cl})\end{array}$ & $\begin{array}{l}p \\
\text { value }\end{array}$ \\
\hline $\begin{array}{l}\text { Quartile } \\
1\end{array}$ & $389 / 2550$ & Ref & & Ref & & Ref & & Ref & \\
\hline $\begin{array}{l}\text { Quartile } \\
2\end{array}$ & $429 / 2550$ & $\begin{array}{l}1.08(0.94- \\
1.24)\end{array}$ & 0.250 & $\begin{array}{l}1.07(0.93- \\
1.23)\end{array}$ & 0.318 & $\begin{array}{l}1.03(0.90- \\
1.19 \bigotimes\end{array}$ & 0.645 & $\begin{array}{l}1.05(0.91- \\
1.21)\end{array}$ & 0.505 \\
\hline $\begin{array}{l}\text { Quartile } \\
3\end{array}$ & $460 / 2547$ & $\begin{array}{l}1.17(1.02 \\
1.34)\end{array}$ & 0.023 & $\begin{array}{l}1.16(1.01- \\
1.33)\end{array}$ & 0.041 & $\begin{array}{l}1.13(0.96- \\
1.33)\end{array}$ & 0.134 & $\begin{array}{l}1.17(0.99- \\
1.38)\end{array}$ & 0.055 \\
\hline $\begin{array}{l}\text { Quartile } \\
4\end{array}$ & $537 / 2549$ & $\begin{array}{l}1.27(1.09- \\
1.18)\end{array}$ & $<0.001$ & $\begin{array}{l}1.14(1.09- \\
1,19)\end{array}$ & $<0.001$ & $\begin{array}{l}1.12(1.06- \\
1.20)\end{array}$ & $<0.001$ & $\begin{array}{l}1.13(1.06- \\
1.20)\end{array}$ & $<0.001$ \\
\hline $\begin{array}{l}\text { Per } 1 \\
\text { SD }\end{array}$ & 1815/10196 & $\begin{array}{l}1.14(1.09- \\
1.19)\end{array}$ & $<0.001$ & $\begin{array}{l}1.16(1.11- \\
1.22)\end{array}$ & $<0.001$ & $\begin{array}{l}1.17(1.09- \\
1.26)\end{array}$ & $<0.001$ & $\begin{array}{l}1.19(1.11- \\
1.28)\end{array}$ & $<0.001$ \\
\hline
\end{tabular}

Major coronary events (CV death, non-fatal MI, or unstable angina)

\begin{tabular}{|c|c|c|c|c|c|c|c|c|c|}
\hline $\begin{array}{l}\text { TyG } \\
\text { index }\end{array}$ & $\begin{array}{l}\text { Events/No. } \\
\text { at risk }\end{array}$ & $\begin{array}{l}\text { Unadjusted } \\
\text { HR (95\% Cl) }\end{array}$ & $\begin{array}{l}p \\
\text { value }\end{array}$ & $\begin{array}{l}\text { Model } 1 \\
\mathrm{HR}(95 \% \\
\mathrm{Cl})\end{array}$ & $\begin{array}{l}p \\
\text { value }\end{array}$ & $\begin{array}{l}\text { Model } 2 \\
\text { HR }(95 \% \\
\mathrm{Cl})\end{array}$ & $\begin{array}{l}p \\
\text { value }\end{array}$ & $\begin{array}{l}\text { Model } 3 \\
\mathrm{HR}(95 \% \\
\mathrm{Cl})\end{array}$ & $\begin{array}{l}p \\
\text { value }\end{array}$ \\
\hline $\begin{array}{l}\text { Quartile } \\
1\end{array}$ & $368 / 2550$ & Ref & & Ref & & Ref & & Ref & \\
\hline $\begin{array}{l}\text { Quartile } \\
2\end{array}$ & $461 / 2550$ & $\begin{array}{l}1.24(1.08- \\
1.43)\end{array}$ & 0.002 & $\begin{array}{l}1.22(1.06- \\
1.40)\end{array}$ & 0.005 & $\begin{array}{l}1.21(1.05- \\
1.40)\end{array}$ & 0.008 & $\begin{array}{l}1.22(1.06- \\
1.41)\end{array}$ & 0.006 \\
\hline $\begin{array}{l}\text { Quartile } \\
3\end{array}$ & $466 / 2547$ & $\begin{array}{l}1.12(1.05- \\
1.20)\end{array}$ & 0.001 & $\begin{array}{l}1.11(1.04- \\
1.19)\end{array}$ & 0.003 & $\begin{array}{l}1.11(1.02- \\
1.20)\end{array}$ & 0.015 & $\begin{array}{l}1.12(1.03- \\
1.22)\end{array}$ & 0.007 \\
\hline $\begin{array}{l}\text { Quartile } \\
4\end{array}$ & $552 / 2549$ & $\begin{array}{l}1.16(1.11- \\
1.21)\end{array}$ & $<0.001$ & $\begin{array}{l}1.16(1.11- \\
1.22)\end{array}$ & $<0.001$ & $\begin{array}{l}1.16(1.09- \\
1.23)\end{array}$ & $<0.001$ & $\begin{array}{l}1.17(1.10- \\
1.24)\end{array}$ & $<0.001$ \\
\hline $\begin{array}{l}\text { Per } 1 \\
\text { SD }\end{array}$ & $1847 / 10196$ & $\begin{array}{l}1.16(1.11- \\
1.21)\end{array}$ & $<0.001$ & $\begin{array}{l}1.17(1.11- \\
1.22)\end{array}$ & $<0.001$ & $\begin{array}{l}1.20(1.12- \\
1.29)\end{array}$ & $<0.001$ & $\begin{array}{l}1.22(1.14- \\
1.31)\end{array}$ & $<0.001$ \\
\hline
\end{tabular}

Model 1: Adjusted for baseline age, sex, previous cardiovascular event, race, BMI, education, systolic blood pressure, and diastolic blood pressure

Model 2: Adjusted for model 1 covariates plus baseline eGFR, HbA1c, total plasma cholesterol, plasma HDL-C, and depression.

Model 3: Adjusted for model 2 covariates plus treatment of statin, biguanide, aspirin, ACEI/ARB, and insulin.

BMI: body mass index; HDL-C: high-density lipoprotein cholesterol; ACEl: angiotensin-converting enzyme inhibitor; ARB: angiotensin receptor blocker; $\mathrm{Cl}$ : confidence interval; HR: hazard ratio; CV: cardiovascular; MI: myocardial infarction; TyG: triglyceride-glucose.

\section{Associations between TyG index trajectories and adverse cardiovascular or major coronary events}


A total of 9697 participants were included in the further trajectory analysis (Fig. 1). Four discrete trajectories with stable TyG indexes at various levels from visit 1 to visit 11 were identified (Fig. 4): low ( $n=1568,16.17 \%)$, moderate ( $n=3,880,40.010 \%)$, high $(n=3,372,34.6 \%)$, and very high ( $n=877,9.3 \%)$ TyG index trajectory groups. The median (interquartile range) changes in TyG index level during the visits for these trajectory groups were $0.009(-0.008-0.02)$ in the low trajectory group, $0.007(-0.02-0.02)$ in the moderate trajectory group, $-0.007(-0.03-0.003)$ in the high trajectory group, $0.007(-0.05-0.05)$ in the very high trajectory group (Tables S3). As shown in Fig. 5, the rates of adverse CV events were $14.16 \%, 16.57 \%, 19.48 \%$, $22.81 \%$, in the low, moderate, high, and very high. TyG index trajectory groups, respectively $(p<0.001)$. In addition, the rates of major coronary events were $12.88 \%, 17.22 \%, 19.63 \%, 25.09 \%$, in the low, moderate, high, and very high. TyG index trajectory groups, respectively $(p<0.001)$. Multivariate Cox regression analyses identified those with TyG index trajectory at high and very high levels as having an even greater risk of adverse CV events in 3 different adjusted models (all $P<0.05$, Table 3). In the fully adjusted model, compared with those with a low trajectory at a low level, the odds ratios (95\% Cls) for associations of those participants with TyG index trajectories at the moderate, high, and very high levels with the risk of adverse CV event were 1.20 (95\% Cl,1.03-1.41; $\mathrm{P}=0.021), 1.25(95 \% \mathrm{Cl}, 1.15-1.37 ; p<0.001)$, and $1.26(95 \% \mathrm{Cl}, 1.16-1.37 ; p<$ 0.001 ), respectively (model 3 in Table 3 ). Results were similar when evaluated the risk of major coronary events for various levels of TyG index trajectory groups.

Table 3. Risk of adverse CV and major coronary events for various levels of TyG index trajectory groups.

Adverse CV events (a composite of CV death, non-fatal MI, or non-fatal stroke)

\begin{tabular}{|c|c|c|c|c|c|c|c|c|}
\hline $\begin{array}{l}\text { TyG index } \\
\text { trajectories }\end{array}$ & $\begin{array}{l}\text { Unadjusted } \\
\text { HR }(95 \% \mathrm{Cl})\end{array}$ & $\begin{array}{l}p \\
\text { value }\end{array}$ & $\begin{array}{l}\text { Model } 1 \\
\text { HR }(95 \% \mathrm{Cl})\end{array}$ & $\begin{array}{l}p \\
\text { value }\end{array}$ & $\begin{array}{l}\text { Model } 2 \\
\text { HR }(95 \% \mathrm{Cl})\end{array}$ & $\begin{array}{l}p \\
\text { value }\end{array}$ & $\begin{array}{l}\text { Model } 3 \\
\text { HR }(95 \% \\
\text { Cl) }\end{array}$ & $\begin{array}{l}p \\
\text { value }\end{array}$ \\
\hline Low & Reference & & Reference & & Reference & & Reference & \\
\hline Moderate & $\begin{array}{l}1.21 \otimes 1.04- \\
1.41 \rrbracket\end{array}$ & 0.015 & $\begin{array}{l}1.21 \otimes 1.03- \\
1.40 \rrbracket\end{array}$ & 0.018 & $\begin{array}{l}1.20 \bigotimes 1.03- \\
1.41 \rrbracket\end{array}$ & 0.022 & $\begin{array}{l}1.20 \otimes 1.03- \\
1.41 \rrbracket\end{array}$ & 0.021 \\
\hline High & $\begin{array}{l}1.22 \bigotimes 1.13- \\
1.32 \bigotimes\end{array}$ & $<0.001$ & $\begin{array}{l}1.23 \otimes 1.14- \\
1.33 \rrbracket\end{array}$ & $<0.001$ & $\begin{array}{l}1.25 \bigotimes 1.15- \\
1.36 \rrbracket\end{array}$ & $<0.001$ & $\begin{array}{l}1.25 \rrbracket 1.15- \\
1.37 \rrbracket\end{array}$ & $<0.001$ \\
\hline Very high & $\begin{array}{l}1.22 \bigotimes 1.14- \\
1.30 \bigotimes\end{array}$ & $<0.001$ & $\begin{array}{l}1.24 \otimes 1.16- \\
1.33 \rrbracket\end{array}$ & $<0.001$ & $\begin{array}{l}1.25 \rrbracket 1.15- \\
1.35 \rrbracket\end{array}$ & $<0.001$ & $\begin{array}{l}1.26 \rrbracket 1.16- \\
1.37 \rrbracket\end{array}$ & $<0.001$ \\
\hline
\end{tabular}

Major coronary events (CV death, non-fatal MI, or unstable angina)

\begin{tabular}{|c|c|c|c|c|c|c|c|c|}
\hline $\begin{array}{l}\text { TyG index } \\
\text { trajectories }\end{array}$ & $\begin{array}{l}\text { Unadjusted } \\
\text { HR (95\% Cl) }\end{array}$ & $\begin{array}{l}p \\
\text { value }\end{array}$ & $\begin{array}{l}\text { Model } 1 \\
\text { HR }(95 \% \mathrm{Cl})\end{array}$ & $\begin{array}{l}p \\
\text { value }\end{array}$ & $\begin{array}{l}\text { Model } 2 \\
\text { HR }(95 \% \mathrm{Cl})\end{array}$ & $\begin{array}{l}p \\
\text { value }\end{array}$ & $\begin{array}{l}\text { Model } 3 \\
\text { HR (95\% } \\
\mathrm{Cl})\end{array}$ & $\begin{array}{l}p \\
\text { value }\end{array}$ \\
\hline Low & Reference & & Reference & & Reference & & Reference & \\
\hline Moderate & $\begin{array}{l}1.40(1.19- \\
1.63)\end{array}$ & $<0.001$ & $\begin{array}{l}1.37(1.17- \\
1.60)\end{array}$ & $<0.001$ & $\begin{array}{l}1.39(1.18- \\
1.63)\end{array}$ & $<0.001$ & $\begin{array}{l}1.38(1.18- \\
1.63)\end{array}$ & $<0.001$ \\
\hline High & $\begin{array}{l}1.28(1.18- \\
1.39)\end{array}$ & $<0.001$ & $\begin{array}{l}1.29(1.19- \\
1.40)\end{array}$ & $<0.001$ & $\begin{array}{l}1.31(1.20- \\
1.43)\end{array}$ & $<0.001$ & $\begin{array}{l}1.32(1.21- \\
1.44)\end{array}$ & $<0.001$ \\
\hline Very high & $\begin{array}{l}1.30(1.22- \\
1.38)\end{array}$ & $<0.001$ & $\begin{array}{l}1.31(1.22- \\
1.41)\end{array}$ & $<0.001$ & $\begin{array}{l}1.33(1.23- \\
1.45)\end{array}$ & $<0.001$ & $\begin{array}{l}1.34(1.24- \\
1.46)\end{array}$ & $<0.001$ \\
\hline
\end{tabular}

Model 1: Adjusted for baseline age, sex, previous cardiovascular event, race, BMI, education, systolic blood pressure, and diastolic blood pressure 
Model 2: Adjusted for model 1 covariates plus baseline eGFR, HbA1c, total plasma cholesterol, plasma HDL-C, and depression.

Model 3: Adjusted for model 2 covariates plus treatment of statin, biguanide, aspirin, ACEI/ARB, and insulin.

BMI: body mass index; HDL-C: high-density lipoprotein cholesterol; ACEl: angiotensin-converting enzyme inhibitor; ARB: angiotensin receptor blocker; Cl: confidence interval; HR: hazard ratio,; CV: cardiovascular; Ml: myocardial infarction; TyG: triglyceride-glucose.

\section{Discussion}

In this large-scale, prospective cohort study, we have evaluated the impact of the TyG index on incident CV events in patients with T2DM during 9 years of follow-up. This study assessed the association between the baseline TyG index and the primary as well as secondary adverse CV outcomes, which revealed that higher levels of TyG index are significantly associated with an increased risk of adverse CV events. More precisely, among all the adverse CV events, all-cause death, CV death, non-fatal MI, non-fatal stroke, total stroke, fatal or hospital CHF, and major coronary events, the TyG index was strongly associated with major coronary events. Thus, it is suggested that TyG has been closely observed with the formation and development of atherosclerosis and finally showed a significant increase in the incidence of clinical coronary events with a high TyG index. Further, we identified that the four distinct trajectories of the TyG index confer different risks of adverse CV events, and a decade trajectory with elevated TyG index carries a greater risk of future incident adverse CV events. These findings suggest a potential role for a long-lasting high level of TyG in the pathogenesis of CVD. Therefore, frequent screening for adverse CV events and aggressive risk factor management in these patients would be highly beneficial.

CVD, a group of heart and blood vessel disorders, including CHD, stroke, and other conditions, is the leading cause of death worldwide, estimating 17.9 million lives yearly. More than $75 \%$ of deaths due to CVD occur in low- and middle-income countries, and $85 \%$ of all deaths due to CVD are due to heart attacks and strokes[28, 29]. While CVDs are responsible for many deaths globally, IR, a key feature of metabolic syndrome and T2DM, was considered a significant risk factor for $\operatorname{CVD}[28,29]$. However, the insulin concentrations are not routinely measured in clinical settings and are similar in the ACCORD trial. Therefore, the TyG index was used as a biomarker of IR in this post hoc analysis to study its relationship with CVD risk in diabetic patients. Moreover, there is the immense advantage of using the TyG index, including being easily accessible in any clinical setting and making our findings usable. Multiple studies indicated that TyG is an indicator of IR, and there is an association between TyG and increased risk of CVD. Recent studies suggested that IR may account for the mechanism underlying the association of the TyG index with adverse CV outcomes. These studies have indicated the importance of IR in atherogenesis and advanced plaque progression[8, 30, 31]. It has been well-established that IR and coexisting hyperinsulinemia are implicated in the development of dyslipidemia, hypertension, hypercoagulability, and atherosclerosis $[7,8]$. In turn, these metabolic changes caused by IR could promote the development of CVD. In particular, chronic hyperglycemia-induced by IR causes oxidative stress. Subsequently, it triggers an inflammatory response that promotes vascular cell damage. Regarding lipid metabolism, IR leads to elevated plasma triglycerides, reduced plasma HDL$\mathrm{C}$, and the appearance of small dense LDL-C particles [32]. In addition, IR has been demonstrated to be implicated in the decreased fibrinolytic activity and increased thrombotic events [33,34]. It is worth noting that IR could promote atherosclerosis not only through mechanisms that involve systemic factors, such as dyslipidemia, hypertension, and a proinflammatory state, but also through the effect of perturbed insulin signaling at the level of the intimal cells [8,35]. Therefore, further studies are required to elucidate the precise mechanism.

Consequently, several clinical studies were conducted to investigate the association of the TyG index with CVD morbidity and mortality in the general population. However, there is a paucity of prospective data regarding the association between IR assessed by the TyG index and CVD in diabetic patients. Among these studies, a cross-sectional study, including 888 asymptomatic T2DM patients without CHD showed that a higher TyG index was associated with an increased risk of 
significant coronary artery stenosis[36]. Moreover, a nested case-control study of 1282 T2DM patients with stable CAD showed that the TyG index was positively associated with future CV events, defined as all-cause death, non-fatal MI, stroke, and post-discharge revascularization[37]. Additionally, a cohort study of 25,969 participants without previous diabetes or CVD indicated substantial similarities in the inflammatory profiles associated with diabetes and CVD[38]. Recently, a mediation analysis was performed to quantify the magnitude and relative contributions of several traditional or nontraditional CV risk factors in the pathway from T2DM to increased CV events (MI, stroke, and vascular mortality). The study demonstrated that the most important pathway contributing to CV events was the presence of IR assessed by the TyG index, followed by elevated triglycerides, the presence of microalbuminuria, and reduced kidney function. At the same time, the excess risk was not mediated through elevated systolic blood pressure or high LDL-C [39]. Our study showed that the metabolic risk factors such as hypertension, diabetes, and hyperlipidemia, were more obvious among participants of higher quartiles of the TyG index. Meanwhile, individuals with the highest quartile of the baseline TyG index had a 2.23-fold higher risk for adverse CV events than those with the lowest quartile. The association remained statistically significant after adjusting for all the aforementioned CVD risk factors. These findings suggested that the clinical management of the TyG index may bring additional effects on CVD development even under vigorous control of traditional risk factors.

Furthermore, previous studies based on the TyG index measured at a single time point, which may not reflect long-term exposure, for the TyG index levels may vary over time. The participant's electronic medical record allows rapid integration of data across multiple time points. Thus, longitudinal measurements and recording of TyG indexes to identify TyG index trajectories are feasible. It represents an added value to the baseline levels to plan and monitor participants' follow-up. On the other hand, a recent study used the data from the atherosclerosis risk in communities (ARIC) study to evaluate the association of the TyG index with PAD and to determine the influence of baseline TyG index and different trajectories of its change over 20 years on the development of PAD. This suggested that a two-decade trajectory with an elevated TyG index carries a greater risk of future incident PAD [40]. Therefore, measurements of long-term trajectories of the TyG index provide reliable and robust results. Our study assessed the impact of long-lasting IR at various levels for the first time using the TyG index for future adverse CV events in patients with T2DM. Our study revealed that there were heterogeneous patterns of trends in the TyG index within the ACCORD population. The baseline TyG index levels cannot fully depict this dynamic change of trend over time. In addition, risks for individuals may change during follow-up. Thus, the TyG index trajectory reflects the long-term impact of the TyG index on adverse CV outcomes. Besides, our study suggests that those trajectory groups with long-term high and very high TyG index levels are at a greater risk of adverse CV events over 10 years after adjustment for traditional CVD risk factors. We can graph trends in the TyG index to identify high-risk individuals who behave similarly to those with TyG index trajectories at high and very high levels observed in the present analysis. Such a population may benefit from earlier and more frequent screening for adverse CV events and aggressive risk factor management, such as control of blood pressure, cessation of smoking, maintaining of metabolic health.

Nonetheless, this study also has several limitations: (i) We could not exclude the possibility of residual confounders despite our careful adjustment for the well-known and suspected risk factors due to the nature of any observational studies. (ii) Patients included in the study were mainly Caucasians aged 40-79 years at baseline. Thus, it may differ outside this age range and in other ethnicities. (iii) We could not compare trajectories of the TyG index with HOMA-IR for predicting adverse cardiovascular events due to the missing records of insulin levels in the ACCORD/ACCORDION study. Despite these limitations that might interfere with the clinical application of the TyG threshold found in our study, it has shown that it is necessary to strictly monitor lipid and glucose levels among T2DM patients during the long-term follow-up.

\section{Conclusion}

TyG index was significantly and positively associated with adverse CV outcomes, suggesting that the TyG index may be a valuable predictor of adverse CV outcomes in patients with T2DM. More importantly, long-term trajectories of the TyG index identify individuals at a higher risk of adverse CV events in patients with T2DM who deserve specific preventive and therapeutic approaches.

Page 12/19 


\section{Declarations}

\section{Acknowledgements}

We thank all participants and ACCORD/ACCORDION investigators for their tremendous support in the trial. We also thank the Core Facility at the National Clinical Research Center for Metabolic Diseases for interpretation of results.

\section{Conflicts of interest}

The authors declare that they have no conflicts of interest.

\section{Funding}

This research was supported by the National Natural Science Foundation of China (81801394 to Shi Tai) and the Natural Science Foundation of Hunan Province (2019JJ50878 to Shi Tai).

\section{Availability of data and material}

The datasets used and analyzed during the current study are available from the ACCORD/ACCORDION Research Materials obtained from the National Heart, Lung, and Blood Institute (NHLBI) Biologic Specimen and Data Repository Information Coordinating Center. The contents of this report do not necessarily reflect the opinions or views of the ACCORD/ACCORDION study authors or the NHLBI.

\section{Code availability}

Not applicable

\section{Authors' contributions}

This study was completed in collaboration with the following authors: SZ and ST defined the study theme and methods. LF, NZ, and YZ analyzed the data. LF wrote the paper. JS, ZX, ST, and YW edited the paper. All authors read and approved the final manuscript.

\section{Ethics approval}

Not applicable

\section{Consent to participate}

Not applicable

\section{Consent for publication}

All the authors listed above approved the manuscript for publication.

\section{References}

1. American Diabetes A. 10. Cardiovascular Disease and Risk Management: Standards of Medical Care in Diabetes-2019. Diabetes Care. 2019;42(Suppl 1):103-23.

2. Beckman JA, Paneni F, Cosentino F, Creager MA. Diabetes and vascular disease: pathophysiology, clinical consequences, and medical therapy: part II. Eur Heart J. 2013;34(31):2444-52.

3. Booth GL, Kapral MK, Fung K, Tu JV. Relation between age and cardiovascular disease in men and women with diabetes compared with non-diabetic people: a population-based retrospective cohort study. Lancet. 
2006;368(9529):29-36.

4. Kumar A, Singh V. Atherogenic dyslipidemia and diabetes mellitus: what's new in the management arena? Vasc Health Risk Manag. 2010;6:665-9.

5. Guariguata L, Whiting DR, Hambleton I, Beagley J, Linnenkamp U, Shaw JE. Global estimates of diabetes prevalence for 2013 and projections for 2035. Diabetes Res Clin Pract. 2014;103(2):137-49.

6. DeFronzo RA. Insulin resistance, lipotoxicity, type 2 diabetes and atherosclerosis: the missing links. The Claude Bernard Lecture 2009. Diabetologia. 2010;53(7):1270-87.

7. Wang T, Zhao Z, Xu Y, Qi L, Xu M, Lu J, Li M, Chen Y, Dai M, Zhao W, et al. Insulin Resistance and beta-Cell Dysfunction in Relation to Cardiometabolic Risk Patterns. J Clin Endocrinol Metab. 2018;103(6):2207-15.

8. Bornfeldt KE, Tabas I. Insulin resistance, hyperglycemia, and atherosclerosis. Cell Metab. 2011;14(5):575-85.

9. Matthews DR, Hosker JP, Rudenski AS, Naylor BA, Treacher DF, Turner RC. Homeostasis model assessment: insulin resistance and beta-cell function from fasting plasma glucose and insulin concentrations in man. Diabetologia. 1985;28(7):412-9.

10. Bonora E, Targher G, Alberiche M, Bonadonna RC, Saggiani F, Zenere MB, Monauni T, Muggeo M. Homeostasis model assessment closely mirrors the glucose clamp technique in the assessment of insulin sensitivity: studies in subjects with various degrees of glucose tolerance and insulin sensitivity. Diabetes Care. 2000;23(1):57-63.

11. Muniyappa R, Lee S, Chen H, Quon MJ. Current approaches for assessing insulin sensitivity and resistance in vivo: advantages, limitations, and appropriate usage. Am J Physiol Endocrinol Metab. 2008;294(1):E15-26.

12. Guerrero-Romero F, Simental-Mendia LE, Gonzalez-Ortiz M, Martinez-Abundis E, Ramos-Zavala MG, HernandezGonzalez SO, Jacques-Camarena O, Rodriguez-Moran M. The product of triglycerides and glucose, a simple measure of insulin sensitivity. Comparison with the euglycemic-hyperinsulinemic clamp. J Clin Endocrinol Metab. 2010;95(7):3347-51.

13. Ma X, Dong L, Shao Q, Cheng Y, Lv S, Sun Y, Shen H, Wang Z, Zhou Y, Liu X. Triglyceride glucose index for predicting cardiovascular outcomes after percutaneous coronary intervention in patients with type 2 diabetes mellitus and acute coronary syndrome. Cardiovasc Diabetol. 2020;19(1):31.

14. Simental-Mendia LE, Rodriguez-Moran M, Guerrero-Romero F. The product of fasting glucose and triglycerides as surrogate for identifying insulin resistance in apparently healthy subjects. Metab Syndr Relat Disord. 2008;6(4):299304.

15. Sanchez-Inigo L, Navarro-Gonzalez D, Fernandez-Montero A, Pastrana-Delgado J, Martinez JA. The TyG index may predict the development of cardiovascular events. Eur J Clin Invest. 2016;46(2):189-97.

16. Park K, Ahn CW, Lee SB, Kang S, Nam JS, Lee BK, Kim JH, Park JS. Elevated TyG Index Predicts Progression of Coronary Artery Calcification. Diabetes Care. 2019;42(8):1569-73.

17. Li S, Guo B, Chen H, Shi Z, Li Y, Tian Q, Shi S. The role of the triglyceride (triacylglycerol) glucose index in the development of cardiovascular events: a retrospective cohort analysis. Sci Rep. 2019;9(1):7320.

18. Alizargar J, Bai CH. Comparison of Carotid Ultrasound Indices and the Triglyceride Glucose Index in Hypertensive and Normotensive Community-Dwelling Individuals: A Case Control Study for Evaluating Atherosclerosis. Medicina (Kaunas) 2018, 54(5).

19. da Silva A, Caldas APS, Hermsdorff HHM, Bersch-Ferreira AC, Torreglosa CR, Weber B, Bressan J. Triglyceride-glucose index is associated with symptomatic coronary artery disease in patients in secondary care. Cardiovasc Diabetol. 2019;18(1):89.

20. Jin JL, Cao YX, Wu LG, You XD, Guo YL, Wu NQ, Zhu CG, Gao Y, Dong QT, Zhang HW, et al. Triglyceride glucose index for predicting cardiovascular outcomes in patients with coronary artery disease. J Thorac Dis. 2018;10(11):6137-46.

21. Mao Q, Zhou D, Li Y, Wang Y, Xu SC, Zhao XH. The Triglyceride-Glucose Index Predicts Coronary Artery Disease Severity and Cardiovascular Outcomes in Patients with Non-ST-Segment Elevation Acute Coronary Syndrome. Dis Markers.

Page 14/19 
2019;2019:6891537.

22. Luo E, Wang D, Yan G, Qiao Y, Liu B, Hou J, Tang C. High triglyceride-glucose index is associated with poor prognosis in patients with acute ST-elevation myocardial infarction after percutaneous coronary intervention. Cardiovasc Diabetol. 2019;18(1):150.

23. Action to Control Cardiovascular Risk in Diabetes. Study G, Gerstein HC, Miller ME, Byington RP, Goff DC Jr, Bigger JT, Buse JB, Cushman WC, Genuth S, Ismail-Beigi F, et al: Effects of intensive glucose lowering in type 2 diabetes. N Engl J Med 2008, 358(24):2545-59.

24. Group AS, Gerstein HC, Miller ME, Genuth S, Ismail-Beigi F, Buse JB, Goff DC Jr, Probstfield JL, Cushman WC, Ginsberg $\mathrm{HN}$, et al. Long-term effects of intensive glucose lowering on cardiovascular outcomes. N Engl J Med.

2011;364(9):818-28.

25. Nagin DS, Odgers CL. Group-based trajectory modeling in clinical research. Annu Rev Clin Psychol. 2010;6:109-38.

26. Group AS. Nine-Year Effects of 3.7 Years of Intensive Glycemic Control on Cardiovascular Outcomes. Diabetes Care. 2016;39(5):701-8.

27. Group AS, Buse JB, Bigger JT, Byington RP, Cooper LS, Cushman WC, Friedewald WT, Genuth S, Gerstein HC, Ginsberg $\mathrm{HN}$, et al. Action to Control Cardiovascular Risk in Diabetes (ACCORD) trial: design and methods. Am J Cardiol. 2007;99(12A):21i-33i.

28. Laakso M, Kuusisto J. Insulin resistance and hyperglycaemia in cardiovascular disease development. Nat Rev Endocrinol. 2014;10(5):293-302.

29. Adeva-Andany MM, Martinez-Rodriguez J, Gonzalez-Lucan M, Fernandez-Fernandez C, Castro-Quintela E. Insulin resistance is a cardiovascular risk factor in humans. Diabetes Metab Syndr. 2019;13(2):1449-55.

30. Di Pino A, DeFronzo RA. Insulin Resistance and Atherosclerosis: Implications for Insulin-Sensitizing Agents. Endocr Rev. 2019;40(6):1447-67.

31. Reardon CA, Lingaraju A, Schoenfelt KQ, Zhou G, Cui C, Jacobs-El H, Babenko I, Hoofnagle A, Czyz D, Shuman H, et al. Obesity and Insulin Resistance Promote Atherosclerosis through an IFNgamma-Regulated Macrophage Protein Network. Cell Rep. 2018;23(10):3021-30.

32. Ormazabal V, Nair S, Elfeky O, Aguayo C, Salomon C, Zuniga FA. Association between insulin resistance and the development of cardiovascular disease. Cardiovasc Diabetol. 2018;17(1):122.

33. Calles-Escandon J, Mirza SA, Sobel BE, Schneider DJ. Induction of hyperinsulinemia combined with hyperglycemia and hypertriglyceridemia increases plasminogen activator inhibitor 1 in blood in normal human subjects. Diabetes. 1998;47(2):290-3.

34. Sobel BE. Insulin resistance and thrombosis: a cardiologist's view. Am J Cardiol. 1999;84(1A):37J-41J.

35. Giacco F, Brownlee M. Oxidative stress and diabetic complications. Circ Res. 2010;107(9):1058-70.

36. Lee EY, Yang HK, Lee J, Kang B, Yang Y, Lee SH, Ko SH, Ahn YB, Cha BY, Yoon KH, et al. Triglyceride glucose index, a marker of insulin resistance, is associated with coronary artery stenosis in asymptomatic subjects with type 2 diabetes. Lipids Health Dis. 2016;15(1):155.

37. Jin JL, Sun D, Cao YX, Guo YL, Wu NQ, Zhu CG, Gao Y, Dong QT, Zhang HW, Liu G, et al. Triglyceride glucose and haemoglobin glycation index for predicting outcomes in diabetes patients with new-onset, stable coronary artery disease: a nested case-control study. Ann Med. 2018;50(7):576-86.

38. Bao X, Borne Y, Johnson L, Muhammad IF, Persson M, Niu K, Engstrom G. Comparing the inflammatory profiles for incidence of diabetes mellitus and cardiovascular diseases: a prospective study exploring the 'common soil' hypothesis. Cardiovasc Diabetol. 2018;17(1):87.

39. Sharif S, Groenwold RHH, van der Graaf Y, Berkelmans GFN, Cramer MJ, Visseren FLJ, Westerink J, group Ss. Mediation analysis of the relationship between type 2 diabetes and cardiovascular events and all-cause mortality: Findings from the SMART cohort. Diabetes Obes Metab. 2019;21(8):1935-43.

Page 15/19 
40. Gao JW, Hao QY, Gao M, Zhang K, Li XZ, Wang JF, Vuitton DA, Zhang SL, Liu PM. Triglyceride-glucose index in the development of peripheral artery disease: findings from the Atherosclerosis Risk in Communities (ARIC) Study. Cardiovasc Diabetol. 2021;20(1):126.

\section{Figures}

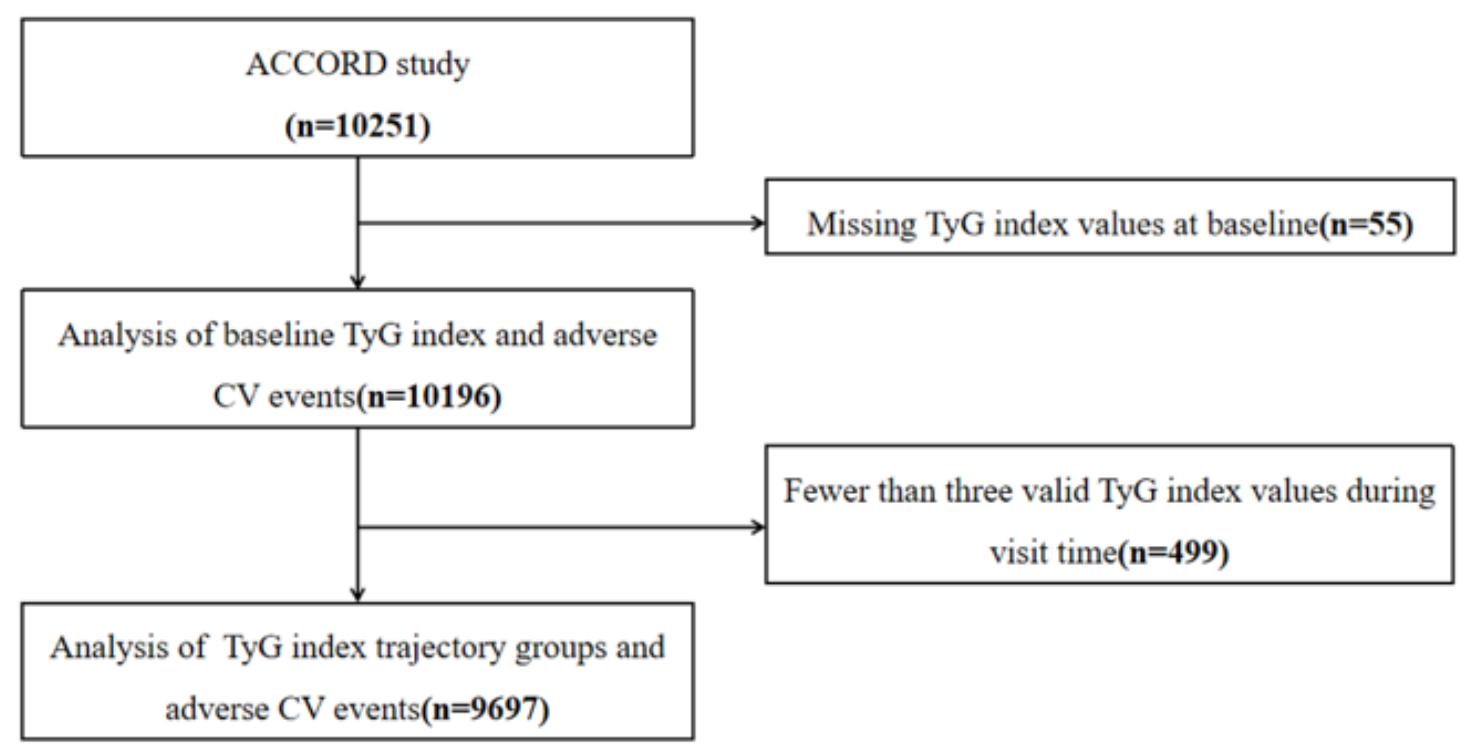

\section{Figure 1}

Flowchart for selecting the Action to Control Cardiovascular Risk in Diabetes (ACCORD) study participants for analysis. Enrollment chart demonstrating patients included in the final sample; ACCORD: Action to Control Cardiovascular Risk in Diabetes; TyG: the triglyceride-glucose; CV: Cardiovascular. 

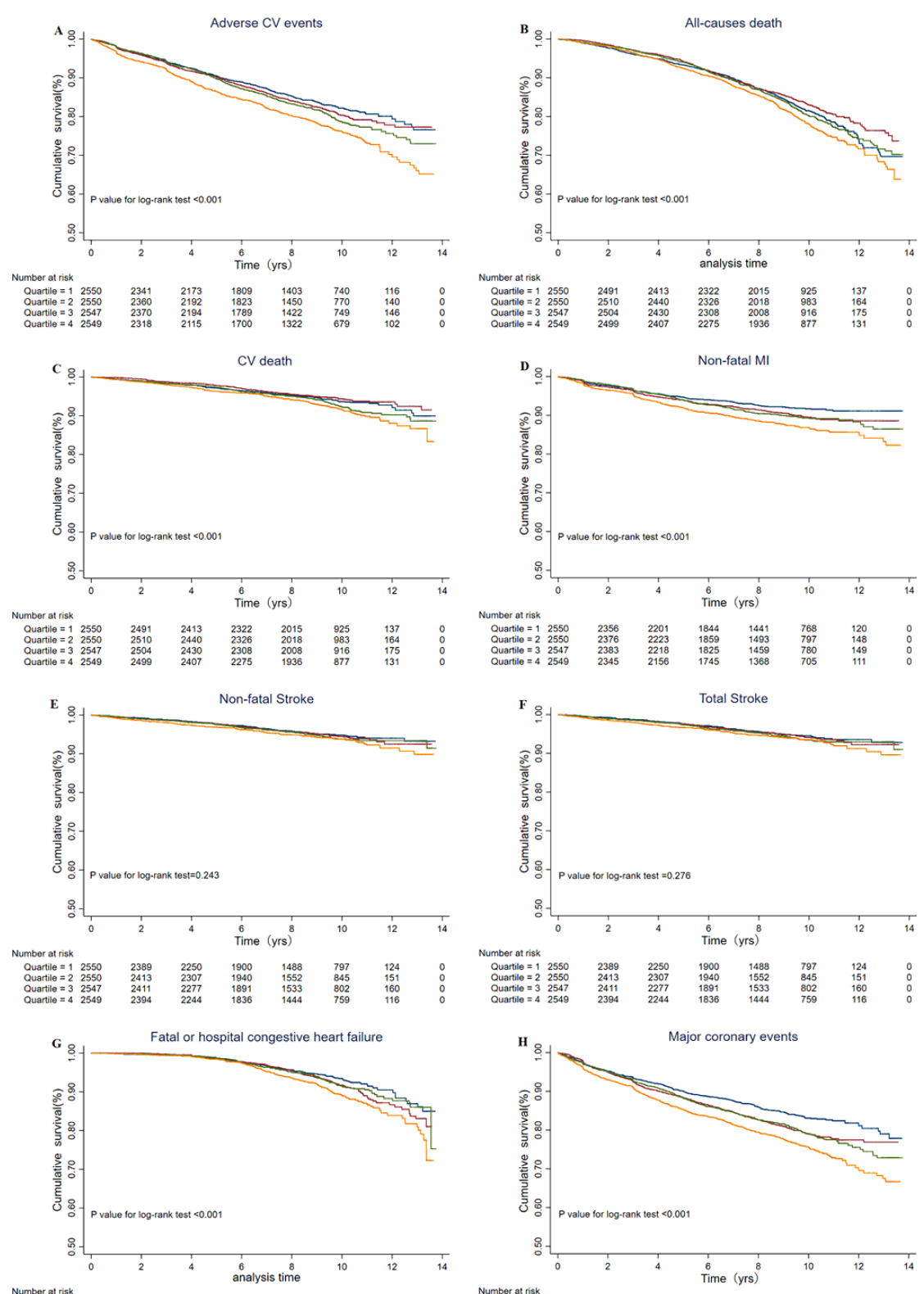

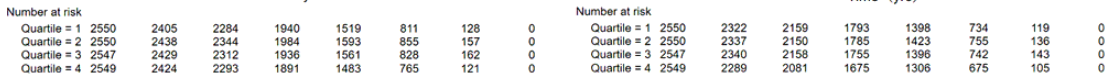

Quartile $=1 \quad$ Quartile $=2$
Quartile $=3 \quad$ Quartile $=4$

\section{Figure 2}

Kaplan-Meier survival curves for primary and secondary outcomes by quartiles of baseline TyG index. (A)Adverse CV events; (B) All-cause death; (C) CV death; (D) Non-fatal MI; (E) Non-fatal stroke; (F) Total stroke; (G) Fatal or hospital congestive heart failure; $(\mathrm{H})$ Major coronary events. CV: Cardiovascular; MI: myocardial infarction. 

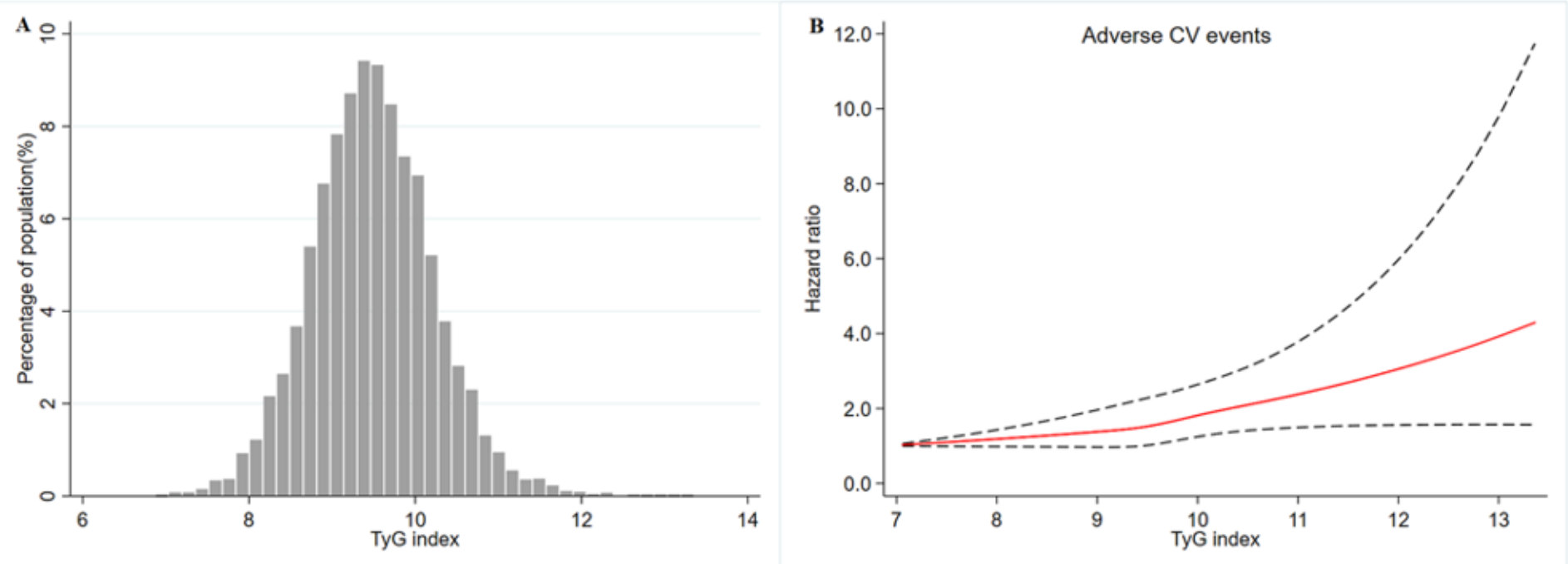

Figure 3

Adjusted hazard ratios of primary outcome by baseline TyG index. (A) Histograms represent the frequency distribution of baseline TyG index; (B) The hazard ratio was adjusted for baseline age, sex, previous cardiovascular event, race, BMI, education, systolic blood pressure, diastolic blood pressure, eGFR, HbA1c, total plasma cholesterol, plasma HDL-C, depression, statin, biguanide, aspirin, ACEI/ARB, and insulin. Red solid line represents the hazard ratio of TyG index across the whole range. Gray dotted lines represent the $95 \%$ Confidence interval.

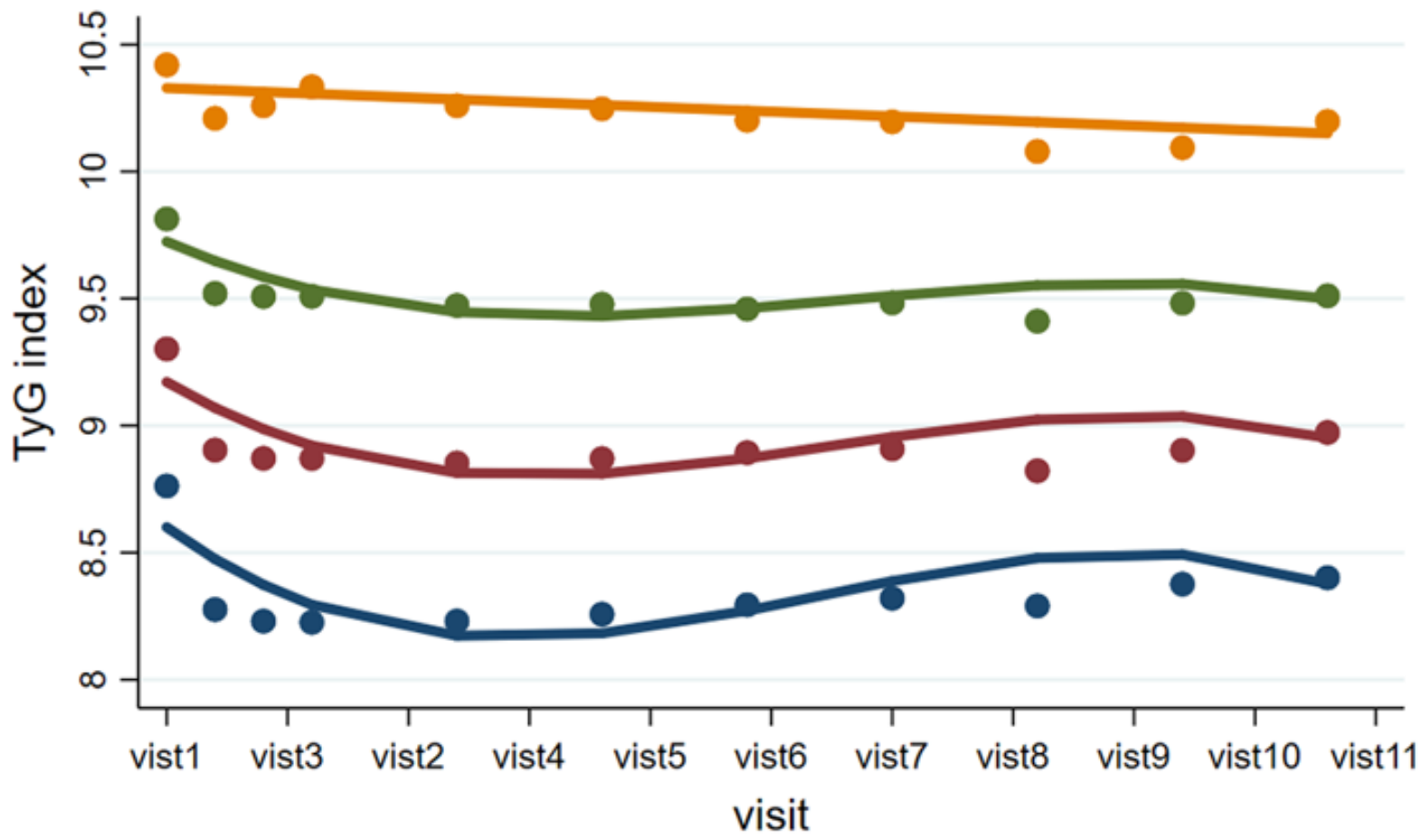

Figure 4 
TyG index trajectory groups and percentage of the population in the group. Four discrete trajectories with stable TyG indexes at various levels from visit 1 to visit 11: low $(n=1568,16.17 \%)$, moderate $(n=3,880,40.010 \%)$, high $(n=3,372,34.6 \%)$, and very high $(n=877,9.3 \%)$ TyG index trajectory groups.
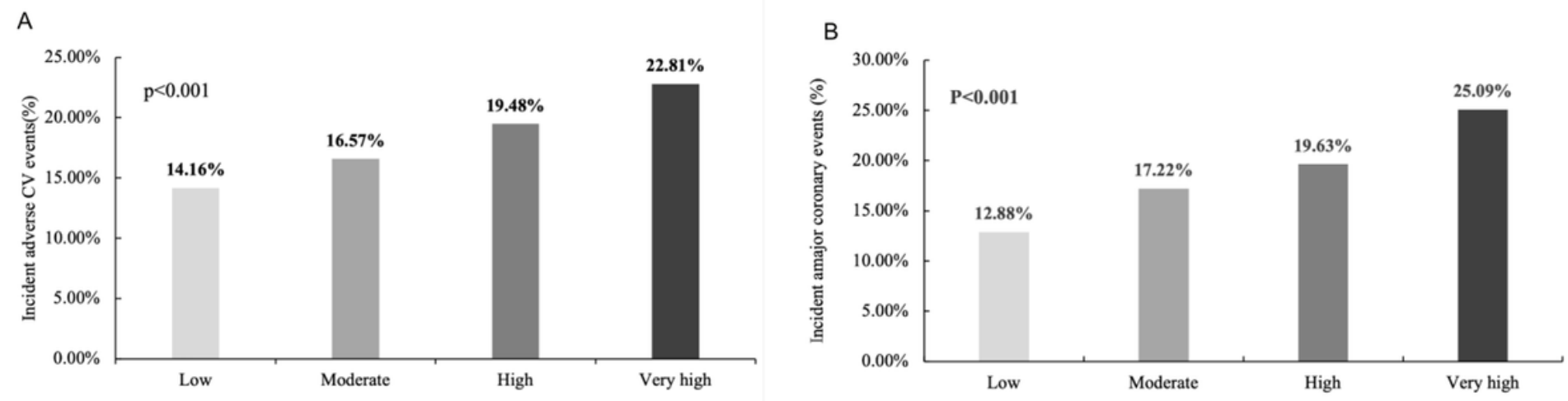

\section{Figure 5}

Prevalence of cardiovascular events across the TyG index trajectory groups. (A) Prevalence of adverse cardiovascular events across the TyG index trajectory groups; (B) Prevalence of major coronary events across the TyG index trajectory groups.

\section{Supplementary Files}

This is a list of supplementary files associated with this preprint. Click to download.

- SupplementryTables.docx 